\title{
FACTORES DE RIESGO PARA ADQUIRIR LAS INFECCIONES DE TRANSMISIÒN SEXUAL EN LOS ADOLESCENTES DEL "COLEGIO MUNICIPAL TÉCNICO SIGLO XXI”. SALINAS. 2013 - 2014
}

\section{RISK FACTORS TO ACQUIRE SEXUAL TRANSMISSION INFECTIONS IN ADOLESCENTS OF THE "COLEGIO MUNICIPAL TÉCNICO SIGLO XXI”. SALINAS. 2013 - 2014}

\author{
Carmen Lascano Espinoza, M.Sc. \\ Sonnia Santos Holguín, M.Sc. \\ Doris Castillo Tomalá, M.Sc. \\ Carrera de Enfermería, Universidad Estatal Península de Santa Elena, Ecuador. cle71@hotmail.com
}

\section{RESUMEN}

El siguiente trabajo de investigación tiene como objetivo determinar el grado de conocimiento sobre los factores de riesgo y las medidas de prevención que tienen los adolescentes del Colegio Técnico Municipal Siglo XXI de la parroquia José Luis Tamayo del Cantón Salinas. La investigación emplea una metodología de diseño cuantitativa, tipo descriptiva y de corte transversal. Para recolectar la información se aplicó un cuestionario a 145 estudiantes del bachillerato, a quienes se valoró la intervención educativa. Entre los resultados obtenidos se evidencia que los adolescentes tienen un déficit en el conocimiento sobre los factores de riesgo conductuales de las Infecciones de Transmisión Sexual (ITS), tales como: uso correcto del preservativo femenino y masculino, prácticas sexuales de riesgo, múltiples parejas sexuales, consumo de bebidas alcohólicas. También se constató que tanto la abstinencia sexual como el contacto directo con personas que manifiesten dermatitis o lesiones en la piel, no son las únicas estrategias para prevenir las ITS. Asimismo, se destaca la importancia de que se imparta la materia de educación para la sexualidad, puesto que no es suficiente la información que se difunde en cualquier medio de comunicación. En general, la mayoría de los jóvenes inician su actividad sexual durante la adolescencia, por consiguiente, el incremento de infecciones constituye un problema social. De ahí la necesidad de implementar una guía educativa acerca de la sexualidad e Infecciones de Transmisión Sexual, y utilizarlo como un recurso didáctico durante el proceso de enseñanza - aprendizaje.

Palabras claves: Factor de riesgo, infecciones de trasmisión sexual.

\section{ABSTRACT}

The following research aims to determine the degree of knowledge about risk factors and preventive measures that adolescents have of the Municipal Technical Colegio Siglo XXI of the parish José Luis Tamayo del Cantón Salinas. The research uses a methodology of quantitative design, descriptive and crosssectional type. To collect the information, a questionnaire was applied to 145 high school students, in which the educational intervention was evaluated. Among the results obtained, it is evident that adolescents have a deficit in knowledge about the behavioral risk factors of Sexually Transmitted Infections, such as: correct use of female and male condoms, risky sexual practices, multiple sexual partners, alcoholic drinks. It was also found that both sexual abstinence and direct contact with people who exhibit dermatitis or skin lesions are not the only strategies to prevent STIs. Likewise, the importance of the education of sexuality is emphasized, since the information disseminated in any medium of communication is not enough. In general, the majority of young people begin their sexual activity during adolescence, therefore, the increase of infections is a social problem. Hence the need to implement an educational guide about sexuality and Sexually Transmitted Infections, and use it as a didactic recruiter during the teaching - learning process.

Key words: Risk factor, sexually transmitted infections.

Recibido: 30 de septiembre de 2016

Aceptado: 20 de marzo de 2017

Publicado: 30 de junio de 2017 


\section{INTRODUCCIÓN}

Las Infecciones de Transmisión Sexual son un problema grave de salud pública a nivel mundial y nacional es muy importante determinar las causas y los factores de riesgo de estas patologías. Las ITS están afectando con mayor intensidad a la población adolescente razón por la cual se ha incrementado en las últimas décadas (1).

Ecuador registra índices muy altos de adolescentes infectados por las infecciones de Transmisión Sexual (ITS). En nuestro país 9 de cada 10 adolescentes son portadores de las ITS, situación semejante a los países del primer mundo, que tiene como factor importante la desinformación sobre la sexualidad y por ende sobre la ITS. Es por esta situación que el Ministerio de Salud Pública implementa el programa de las Enfermedades de Transmisión Sexual (2).

Otro de los factores de las ITS, es a través de los medios de comunicación hablada, escrita y el internet utilizan el sexo como estrategia comercial; lo que ha inducido a los adolescentes a involucrarse en la actividad sexual a temprana edad, y a esto se agrega la falta de responsabilidad y conocimiento de la sexualidad, todos estos factores, motivaron a realizar una investigación del tema. Cuyos resultados revelan que un promedio del $51 \%$ de adolescentes tiene un déficit en el conocimiento de los factores de riesgo conductuales de las Infecciones de Transmisión Sexual lo que da origen a incrementar el índice de morbilidad en la adolescencia.

Por este motivo se plantea implementar una Guía Didáctica dirigida a los adolescentes del Colegio Técnico Municipal Siglo XXI, de la parroquia José Luis Tamayo, del Cantón Salinas, de la provincia de Santa Elena, el mismo que contiene tres módulos: Los factores de riesgo proporcionarán conocimientos sobre factores de riesgo conductuales, pisco-sociales e institucionales de las Infecciones de Transmisión Sexual; las Medidas de prevención permitirán la sensibilización de los diferentes medios de comunicación y finalmente las Enfermedades de Transmisión Sexual que van a fortalecer el conocimiento de las principales infecciones, la sintomatología, las complicaciones y las formas de como transmitirlas.

\section{La adolescencia y la teoría del autocuidado}

La adolescencia, tanto para el hombre como para la mujer, determinan el inicio de una etapa de la vida cargada de nuevas emociones, experiencias y cambios que no sólo establecen la necesidad de una adaptación psicológica, física y social que permitan una mejor integración a esta etapa de la vida, sino que, además, estos cambios constituyen un reto biológico - emocional que es, constantemente, origen de dificultades (3).

La adolescencia es una etapa transitoria de muchos cambios drásticos que significan crecer y desarrollarse hacia la madurez.

Etimológicamente, adolescencia proviene del latín ad: a, hacia, y olescere, de olere: crecer. Significa la condición y el proceso de crecimiento. La adolescencia se define como la etapa del desarrollo humano que tiene un comienzo y una duración variables, la cual marca el final de la niñez y crea los cimientos para la edad adulta. Marcada por cambios interdependientes en las dimensiones biológicas, psicológicas y sociales del ser humano. Desde aproximadamente los 10 hasta los 19 años, los niños sufren cambios corporales, intelectuales y afectivos que generan una tensión lógica por la rápida adaptación a la transición niño - adolescente. No obstante, la gran mayoría realizan una correcta adaptación, superan el suceso satisfactoriamente.

Esta etapa se inicia con los cambios puberales tanto en el hombre como en la mujer. El proceso de los cambios físicos que experimentan durante la pubertad se caracteriza por cinco aspectos íntimamente relacionados, expresados a continuación:

- Rápido aumento en la talla.

- Maduración de los caracteres sexuales primarios.

- Aparición de los caracteres sexuales secundarios.

- Desequilibrio endocrino transitorio.

- Repercusión de todos estos cambios en el área emocional.

La transición es un período que se produce a nivel: Psicológico, se genera una preparación e iniciación a la edad adulta, donde se exhorta el "yo" ante los demás y se encamina una identidad propia. Corporal, adquieren estereotipos de belleza propios de la cultura que son evidentes, 
rápidos y difíciles de asumir. Afectivo, es la formación propia de la identidad que genera la necesidad de amar, pero no ser amado de manera paterno o materno - filial.

Las Infecciones de Transmisión Sexual son aquellas patologías infecto-contagiosas que se transmiten mediante el contacto o coito sexual (oral, vaginal, anal) con una persona infectada, a través de los fluidos corporales. Estas infecciones atacan a cualquier ser humano libremente de la condición socioeconómica, edad, raza, identidad de género y orientación sexual. Las ITS son comunes en adolescentes. La adolescencia se había considerado como una etapa exenta de problemas de salud, pero hoy en día es evidente que los adolescentes enfrentan serios problemas relacionados con la salud sexual, como son las infecciones de transmisión sexual. Las altas tasas de riesgo de salud sexual evidencian que los estilos de vida de la población adolescente son más peligrosos que de la población adulta.

Dorothea Orem describe al ser humano como un organismo biológico, psicológico, en constante interacción con el medio en el que se encuentra; es un ente con gran capacidad de crear, comunicar y realizar las actividades de la vida diaria, beneficiando de manera mutua a los demás. Menciona a la salud como el estado de integridad estructural y funcional que se alcanza mediante las acciones llamadas autocuidados (4).

El autocuidado es acción instruida por los seres humanos que conlleva un objetivo esencial". A su vez, define tres requisitos, objetivos o resultados indispensables para alcanzar el autocuidado e indican una actividad que el individuo debe aplicar para cuidar de sí mismo.

- Requisitos del autocuidado universal. - son actividades comunes para todos los seres humanos e incluyen la conservación del aire, agua, eliminación, ejercicio y descanso, soledad e interacción social, prevención de riesgos e interacción de la actividad humana.

- Requisitos del autocuidado del desarrollo. origina las funciones precisas para la vida y la maduración, aconseja la visión de circunstancias adversas o mitigar los efectos de dichas situaciones en los diversos momentos de proceso evolutivo 0 del desarrollo del ser humano: niñez, adolescencia, adulto y vejez.

- Requisitos del autocuidado de desviación de la salud. - se refiere a situaciones que están vinculadas a los estados de salud.

\section{Factores de riesgo de las Infecciones de Transmisión Sexual}

Factores conductuales: Las Infecciones de Transmisión Sexual influyeron en el siglo XX por medio de los buenos y malos hábitos que hicieron posible la liberación femenina y el libertinaje, siendo la mujer el ente más vulnerable por las siguientes razones: mayor precocidad de actividad sexual, múltiples parejas sexuales, conductas sexuales riesgosas, pérdida de los valores y la desintegración familiar. (5)

Entre los factores de riesgo conductuales se encuentran, el inicio de las actividades sexuales a temprana edad que involucran mayor probabilidad de parejas sexuales durante toda su vida, aumento de las relaciones sexuales con la aprobación de los métodos anticonceptivos, variación de las conductas morales y familiares, diversas parejas sexuales, uso incorrecto del preservativo, practicar sexo sin protección, y consumo de drogas - alcohol asociados a una conducta de riesgo sexual irresponsable. A continuación, se detallan estos factores conductuales:

Prácticas sexuales. El sexo seguro es toda práctica sexual en la que el semen, la sangre o fluidos vaginales de una persona no ingresan al cuerpo de otro individuo, es decir, la relación sexual (abrazos, caricias, masturbación mutua) en la que no existe ningún tipo de penetración (oral, vaginal y anal). El sexo protegido es toda penetración de principio a fin (oral, vaginal y anal) donde se utiliza el preservativo, debido a que se evita el intercambio de fluidos o secreciones, para impedir el contacto con las mucosas. (6).

El uso Correcto del Preservativo, es un método cuya finalidad está en evitar e interrumpir el paso de espermatozoides 0 microorganismos infecciosos al aparato reproductor femenino. Es una funda delgada de plástico de poliuretano que se coloca en la vagina para iniciar y hasta culminar una relación sexual; posee dos anillos flexibles: un anillo interior en el extremo cerrado que se usa para insertar el dispositivo en la vagina y un anillo exterior que se sitúa afuera de la vagina y cubre los genitales externos.

Consumo de bebidas alcohólicas y drogas, el consumo de drogas lícitas e ilícitas con el transcurrir de los días causa dependencia en los seres humanos y dañan su organismo, por otra parte, se afecta el bienestar físico y psicosocial. Se debe tener en cuenta que el alcohol, el tabaco 
o cualquier droga lícita es un impulso para el abuso; el abuso se cataloga como el empleo de frecuentes y altas dosis de cualquier sustancia (alcohol, tabaco, marihuana, etc.). Sin control estas sustancias llevan a la drogadicción causando dependencia, por ende, la ausencia de una droga provoca sentimientos de ansiedad hasta el deseo de consumirla para satisfacer la necesidad. (7).

Múltiples parejas sexuales o conducta sexual, los adolescentes constituyen un grupo vulnerable para las Infecciones de Transmisión Sexual, por esta razón el impartir los conocimientos sobre los factores de riesgo evitará que los estudiantes adquirieran estas patologías. Es así que, cuando existe un mayor número de parejas sexuales, mayor es el riesgo de adquirir las ITS. Los adolescentes deben tomar responsabilidades para comenzar la actividad sexual y llevar una vida sexual a plenitud.

Factores Psicosociales. La adolescencia constituye una etapa evolutiva de los seres humanos, en la cual se manifiestan cambios de inestabilidad psicológica y emocional; unida a importantes contradicciones. También es un período de la vida, que refiere la búsqueda de la propia identidad, siendo muy frecuentes los deseos de experimentación; abarcando los ámbitos de dependencia a sustancias tóxicas y las relaciones sexuales mediante las prácticas de riesgo. Se manifiesta por sentimientos de invulnerabilidad frente a las patologías y eventos negativos expresados por la inexperiencia y carencia cultural, con emociones de autosuficiencia más debilidad para enfrentar y defender decisiones.

Los jóvenes y adolescentes, en la actualidad, presentan una mayor conducta sexual de riesgo que las mujeres debido a su precocidad de iniciar la actividad coital; teniendo en consideración el número de parejas sexuales, el uso infrecuente e inconsciente del preservativo, relaciones sexuales con personas promiscuas y la asociación de aquellas al consumo de drogas. Las diversas características de la personalidad en su contorno guardan relación fundamental con las conductas sexuales riesgosas. (8).

Factores Educativos. El desconocimiento sobre los factores de riesgo de las Infecciones de Transmisión Sexual se enfatiza mediante la falta de educación sexual, de la cual carecen las instituciones educativas. Sin embargo, prevalece aun los tabúes que impiden la impartición de una educación sexual formal, por ende, existen pocos comportamientos de forma audaz en los adolescentes. La OMS manifiesta que la educación sexual no fomenta el aumento al inicio de las relaciones sexuales a tempranas edades por el contrario les retrasa y potencia un mejor uso de los métodos anticonceptivos.

El Ministerio de Salud Pública ha implementado, mediante sus programas educativos en las escuelas y colegios conocimientos sobre el VIH/SIDA, pero esta información es deficiente para los estudiantes debido a la falta de educación sexual para referirse al conjunto de las ITS. La educación es el elemento primordial para que los adolescentes adquieran conocimientos de forma concreta y veraz sobre las ITS; pero es necesario cambiar el paradigma de que, educación sexual es brindar conceptos sobre anatomía humana, reproducción y enfermedades transmisibles dejando a un lado los aspectos relacionados y éticos de la conducta sexual. Es fundamental la educación emocional adjunta a la educación sexual.

\section{Infecciones de trasmisión sexual}

La Organización Mundial de la Salud ha clarificado la denominación de Enfermedades de Transmisión Sexual (ETS) por Infecciones de Transmisión Sexual (ITS) debido a que el término de enfermedad es inadecuado para las infecciones que no expresan manifestación (9). De ahí, la importancia de establecer la diferencia entre infección y enfermedad:

Infección: Los microorganismos (virus, bacterias, hongos 0 parásitos) pueden causar la enfermedad por estar dentro del organismo, pero no presentar sintomatología de infección.

Enfermedad: Expresa la sintomatología de la infección y altera el sistema inmunológico.

La OMS establece que aproximadamente un millón de las ITS corresponden a las infecciones por VIH, agente causal del SIDA; la importancia de estas patologías radica en las complicaciones que las caracterizan (enfermedad pélvica inflamatoria, embarazo ectópico, cáncer cérvidouterino e infertilidad. Las infecciones de transmisión sexual son causadas por un gran número de microorganismos que necesitan lugares húmedos y cálidos para sobrevivir (boca, recto, vagina, vulva, pene y testículos) entre estos tenemos: 
- Bacterias: Neisseria gonorrohoeae, Treponema pallidum, Haemophiluducrey, Gardnerellavaginalis, Clamidias trachomatis

- Virus: Virus de Inmunodeficiencia Humana (VIH), virus de la Hepatitis B, virus del Herpes Simple y virus del papiloma humano.

- Hongos: Cándida albicans.

- Parásitos: Trichomona vaginales, Sarcoptesscaies, Pediculoscorporis y pubis.

\section{MEDIDAS DE PREVENCIÓN DE LAS INFECCIONES DE TRASMISIÓN SEXUAL}

Se debe difundir información responsable y completa sobre este tipo de enfermedades y sus consecuencias físicas, sociales y mortales. Es importante eliminar posibles gérmenes antes de que éstos tengan la oportunidad de infectar. Por ende, se recomienda lavar los genitales con agua, jabón y orinar después de la relación sexual, también utilizar preservativo femenino o masculino para disminuir el riesgo en todos los tipos de prácticas sexuales (vaginal, anal y oral).

Entre las medidas preventivas es necesario evitar el contacto directo ante dermatitis o lesiones en la piel, en las donaciones de sangre y sus hemoderivados es importante la exclusión de personas con factores de riesgo (10).

Entre los consejos apropiados para prevenir las Infecciones de Transmisión Sexual se encuentran: uso correcto del preservativo femenino y masculino, evitar los tipos de relaciones sexuales que aumentan el riesgo de adquirir una ITS (el sexo anal desgarra los tejidos del recto con facilidad), vacuna contra el Virus del Papiloma Humano, acudir a las consultas en el centro de salud para prevenir una ETS, conocer y limitar el número de cada pareja sexual y platicar del consumo de sustancias tóxicas.

\section{OBJETIVOS 2.1.- Objetivo general}

Determinar los factores de riesgo que tienen los adolescentes para adquirir las infecciones de transmisión sexual y qué medidas de prevención deben aplicar los estudiantes del Colegio Municipal Siglo XXI. Salinas 2013 - 2014

\section{2.- Objetivos específicos}

Los objetivos específicos permiten identificar el conocimiento que poseen los estudiantes con relación a los factores de riesgo de las ITS, para su fiel cumplimiento se pretende buscar información sobre las Infecciones de Transmisión Sexual. Diseñar una Guía Educativa que contenga temas relacionados con las enfermedades de transmisión sexual, signos y síntomas, formas de transmisión y medidas de prevención a los adolescentes de la institución. Socializar la Guía Educativa con el fin de que los adolescentes capten mediante una forma apropiada los conocimientos para las Infecciones de Transmisión Sexual. Aplicar la Guía Educativa a los estudiantes - adolescentes de la institución. Evaluar a los adolescentes sobre la información impartida y verificar la adquisición de los conocimientos para bien común.

\section{Materiales y métodos}

El método utilizado en el presente estudio fue de carácter descriptivo, cuantitativo, de corte transversal. Porque buscará la relación entre los factores de riesgo y las medidas de prevención de las infecciones de transmisión sexual en los adolescentes, sin llegar al descubrimiento de relaciones causales entre ellas sino más que todo el grado de relación que guardan. Así mismo se lo ejecutará en un momento determinado y una sola vez la aplicación de encuestas y entrevistas, las mismas que serán proyectadas en cuadros estadísticos, extrayendo las recomendaciones y las sugerencias que contribuyan a la solución de la problemática.

La población es la totalidad de las personas que poseen características comunes susceptibles a ser estudiadas, en este trabajo de investigación se tomó como población a investigar a los estudiantes del bachillerato que se detallan a continuación: 
Tabla 1: Distribución de la población

\begin{tabular}{|c|c|c|c|c|c|c|c|c|}
\hline CURSOS & PARALELOS & $\begin{array}{c}\text { ESTUDIANTES } \\
\text { POR } \\
\text { PARALELOS }\end{array}$ & $\begin{array}{c}\text { TOTAL } \\
\text { ESTUDIANTES }\end{array}$ & PORCENTAJE & $\begin{array}{c}\text { MUESTRA } \\
\text { CALCULADA }\end{array}$ & $\begin{array}{c}\text { MUESTRA } \\
\text { ESTRIFICADA } \\
\text { POR CURSO }\end{array}$ & $\begin{array}{c}\text { PORCENTAJE } \\
\text { POR } \\
\text { PARALELO }\end{array}$ & $\begin{array}{c}\text { NÚMERO } \\
\text { DE } \\
\text { ENCUESTA } \\
\text { POR } \\
\text { PARALELO }\end{array}$ \\
\hline \multirow{3}{*}{$\begin{array}{c}\text { PRIMERO } \\
\text { BACHILLERATO }\end{array}$} & $A$ & 50 & \multirow{3}{*}{149} & \multirow{3}{*}{0,47} & \multirow{9}{*}{145} & \multirow{3}{*}{68} & 0,34 & 23 \\
\hline & B & 49 & & & & & 0,33 & 22 \\
\hline & $\mathrm{C}$ & 50 & & & & & 0,34 & 23 \\
\hline \multirow{3}{*}{$\begin{array}{c}\text { SEGUNDO } \\
\text { BACHILLERATO }\end{array}$} & $A$ & 40 & \multirow{3}{*}{122} & \multirow{3}{*}{0,38} & & \multirow{3}{*}{56} & 0,33 & 18 \\
\hline & B & 42 & & & & & 0,34 & 19 \\
\hline & $C$ & 40 & & & & & 0,33 & 18 \\
\hline \multirow{2}{*}{$\begin{array}{c}\text { TERCERO } \\
\text { BACHILLERATO }\end{array}$} & $\bar{A}$ & 25 & \multirow[b]{2}{*}{47} & \multirow{2}{*}{0,15} & & \multirow[b]{2}{*}{21} & 0,53 & 11 \\
\hline & $B$ & 22 & & & & & 0,47 & 10 \\
\hline TOTAL & 11 & 318 & 318 & 1,00 & & 145 & TOTAL & 145 \\
\hline
\end{tabular}

Instrumento: Estadística de producción del área

\section{RESULTADOS}

Tabla 2: Conocimiento de factores de riesgo de las ITS

\begin{tabular}{|c|c|c|c|}
\hline \multicolumn{2}{|c|}{ Valoración } & Frecuencia & Porcentaje \\
\hline 1 & $\mathrm{Si}$ & 68 & 47 \\
\hline 2 & No & 77 & 53 \\
\hline \multicolumn{2}{|l|}{ Total } & 145 & 100 \\
\hline
\end{tabular}

Instrumento: Encuesta

Según la tabla, indica que el $47 \%$ de los estudiantes tienen un grado de conocimiento con relación a los factores de riesgo de la ITS mientras que el $53 \%$ no los conoce. Se puede determinar que los estudiantes desconocen que algunas ITS se pueden transmitir con el contacto de piel con piel, transfusiones sanguíneas, reutilización de utensilios. Se evidencia que pese a la cantidad de información expuesta en cualquier medio de comunicación, los estudiantes se manifiestan indiferentes, creyendo que es normal tener relaciones sexuales, por lo que se sugiere comprometer a todos los actores de la educación a sensibilizarlos para que difundan una conducta acorde a su edad y así reducir índices de mortalidad.
Tabla 3: Medidas de prevención - Abstinencia Sexual

\begin{tabular}{|c|l|c|c|}
\hline \multicolumn{2}{|c|}{ Valoración } & Frecuencia & $\%$ \\
\hline 1 & $\mathrm{Si}$ & 71 & 49 \\
\hline 2 & No & 74 & 51 \\
\hline \multicolumn{2}{|l|}{ Total } & 145 & 100 \\
\hline
\end{tabular}

Instrumento: Encuesta

La tabla indica que el $51 \%$ de los encuestados no está de acuerdo que la abstinencia es la mejor alternativa para reducir las ITS, mientras que el $49 \%$ si está de acuerdo. Al enamoramiento es un concepto positivo pero no hay que confundir conceptos de amor con placer sexual. Tener sexo implica responsabilidad sobre todo en esta época de tantas infecciones sexuales, y tantos peligros para nuestros adolescentes. Satanizar el sexo, negar el sexo, infundir temor o ignorar su fuerza y poder, es hacer como el avestruz: meter la cabeza en la tierra para no veo la realidad o dar palos a ciegas, mientras el sida hace estragos, entre otras así. Los programas que incluyen mensajes integrales pueden enseñar las habilidades necesarias para practicar la abstinencia, así como proporcionar información a la juventud sexualmente activa respecto a los condones y a la reducción del número de parejas. 
Tabla 4: Asignatura de educación sexual/pensum educativo

\begin{tabular}{|c|c|c|c|}
\hline \multicolumn{2}{|c|}{ Valoración } & Frecuencia & Porcentaje \\
\hline 1 & $\mathrm{Si}$ & 130 & 89.7 \\
\hline 2 & $\mathrm{No}$ & 15 & 10.3 \\
\hline \multicolumn{2}{|l|}{ Total } & 145 & 100.0 \\
\hline
\end{tabular}

Instrumento: Encuesta

En cuanto a la aprobación de implementar la asignatura de Educación Sexual para los estudiantes de bachillerato. La tabla indica que el $89,7 \%$ está de acuerdo y el 10,3\% manifiesta su oposición. En ocasiones resulta duro contemplar cómo determinados programas de educación sexual en centros educativos concretos son parados en seco por un grupo de padres y madres. Habitualmente hay un error de partida en el planteamiento: no se ha tenido en cuenta la opinión de los padres y madres del centro con antelación. Igualmente cierto es que por debajo de los argumentos los padres/madres que desean la paralización de las actividades de Educación Sexual, se encuentra entre otras razones, su propio desconcierto e inhibición ante este tema. Insisto en que esto no tiene base científica porque todos los estudios y la práctica clínica confirman que el mejor remedio a estos problemas, es una verdadera educación sexual. Dicha educación debe tomar en cuenta los valores, el respeto por el otro y los sentimientos. Es fundamental educar con un gran concepto de la responsabilidad, sin meter miedo ni adoctrinar.

\section{CONCLUSIONES}

Luego del análisis e interpretación de los resultados sobre los factores de riesgo para adquirir las Infecciones de Transmisión Sexual, y por estar relacionados con la salud y la educación, se concluye:

Más de la mitad de los adolescentes encuestados no conocen los factores de riesgo conductuales de las ITS, a pesar de que han recibido información sobre los agentes infecciosos. El poco grado de conocimiento que tienen lo han obtenido de forma inequívoca. Por otra parte, los padres se encuentran distantes con respecto a la educación en salud sexual de sus hijos debido a la conservación de los tabúes. Los estudiantes deben capacitarse en medidas de prevención de las Infecciones de Transmisión Sexual (abstinencia sexual, consultas ginecológicas y médicas, fidelidad de la pareja e higiene de los genitales); así como también en la profilaxis de la vacuna del Virus del Papiloma Humano. Es necesario que los estudiantes identifiquen las formas de transmisión de las ITS, la mayoría han presentado cuadros asintomáticos que posteriormente conllevaron a grandes complicaciones en hombres y mujeres después del acto sexual. Algunos encuestados no están satisfechos con el contenido o los programas de educación sexual que reciben en la institución, al parecer hay algunos temas que son de mucha inquietud y no son tomados en cuenta a la hora de enseñar a los estudiantes. Más de la tercera parte de los alumnos considera que se imparta la materia de Educación Sexual, cuyo contenido resalte temas precisos sobre las Infecciones de Transmisión Sexual.

\section{RECOMENDACIONES}

En base al trabajo de investigación realizado, mediante una encuesta; se llegó a las siguientes recomendaciones:

La Institución Educativa debe cumplir con los estándares de calidad y calidez, por lo tanto, debe incluir en el pensum educativo la materia de Educación Sexual, para que los adolescentes adquieran los conocimientos específicos sobre sexualidad y prevención de las Infecciones de Transmisión Sexual. También es fundamental que se coordine un trabajo de equipo con el Ministerio de Salud Pública y los internos de enfermería de la UPSE, para que apliquen la Guía educativa como recurso didáctico que garantice el proceso de enseñanza. Se sugiere a los adolescentes tomen las correctas medidas de prevención para evitar contraer las Infecciones de Transmisión Sexual. Se recomienda a los padres o madres de familia a que realicen las gestiones pertinentes, tales como chequeos médicos, consulta externa, estado de salud, etc. buscando así la detección precoz de las ITS. Además, se recomienda que se involucre a cada miembro de la comunidad educativa para que garantice que el grado de conocimiento sobre los factores de riesgo que predisponen a los adolescentes disminuyen los riesgos de Infecciones de Transmisión Sexual. 


\section{CITAS BIBLIOGRÁFICAS}

(1) BRUZOS C. Educación para la salud en la escuela. 790th ed. Santos Dd, editor. España; 1991.

(2) YEROVI G. Ministerio de Salud Pública. [Online].; 2011 [cited 2016 Julio 12. Available from: http://www.salud.gob.ec/programanacional-de-prevencion-y-control-de-vihsida-its/

(3) UNICEF. Una etapa fundamental. 10017th ed. Nueva York: División de Comunicaciones; 2002.

(4) INMA. Cuidados Críticos y Paliativos. [Online].; 2014 [cited 2012 Octubre 19. Available from:

http://inmaglezglez.wordpress.com/type/aside/

(5) CASTRO ABREU U. Conocimientos y factores de riesgo sobre infecciones de transmisión sexual en adolescentes. 9705716th ed.: Revista Habanera de Ciencias Médicas; 2010.
(6) PANAMÁ E. Opta por una actitud sexual responsable. [Online]; 2011. Available from: Opta por una actitud sexual responsable.

(7) PSICOLOGÍA DEL DESARROLLO. Adultez y Vejez. 7430th ed. Madrid: Médica Panamericana; 2009.

(8) N COLOMBIA. Factores de riesgo para ETS en adolescencia. 1st ed.: Revista de Pediatría; 2012.

(9) CASANOVA R. Infecciones de transmisión sexual. [Online].; 2004 [cited 2012 Agosto $12 . \quad$ Available from: http://www.ebrary.com.

(10) CALDERÓN E. Diagnóstico, tratamiento y prevención de las infecciones de transmisión sexual. 23200th ed. Bogotá: Revista Cubana de Medicina General Integral; 2013. 